\title{
Resumen ejecutivo-traducción news the Alan Guttmacher Institute
}

\author{
Desatender las necesidades de salud reproductiva de las mujeres jóvenes amenaza el progreso global \\ Carencia de educación, anticoncepción y servicios de salud
}

Las mujeres adolescentes alrededor del mundo necesitan un mejor acceso a la educación, a la información sexual y reproductiva y a toda una gama de servicios de salud reproductiva, $\sin$ importar su estado marital o status. Los servicios que necesitan incluyen aquellos sobre anticoncepción, enfermedades de transmisión sexual (ETS), embarazo y crianza a cargo de la mujer. Las 260 millones de mujeres alrededor del mundo entre los 15 y 19 años serán la siguiente generación de madres, trabajadoras y líderes. Para que las mujeres jóvenes puedan llevar a cabo con todo su potencial estos roles, es necesario reconocer su experiencia sexual y satisfacer sus necesidades educativas y de salud reproductiva de acuerdo con el nuevo informe del Instituto Alan Guttmacher "Hacia un Nuevo Mundo": Vida Sexual y Reproductiva de las Mujeres Jóvenes; donde se considera:

Los padres, las comunidades y los gobiernos deben reconocer la rapidez con la que el mundo está cambiando y cuán imperativo es dirigir la atención para mejorar la situación de las niñas y las mujeres jóvenes.

Tenemos hoy la generación más grande de jóvenes en toda la historia, más de un billón de hombres y mujeres entre los 10 y 19 años. Los 541 millones de mujeres jóvenes, de las cuales 281 millones están en la edad de 10 a 14 años -son una gran fuerza demográfica y su modelo de crianza tiene grandes implicaciones sobre el tamaño de la población mundial. Este nuevo informe trae información comparada sobre 53 países desarrollados y en desarrollo y cubre cinco importantes regiones- Asia, América Latina y el Caribe, Africa del Norte y el Medio-Oriente, el sub-Sahara Africano y Europa Occidental, los Estados Unidos y Japón, que representan el 75\% de la población mundial.

Educación: Las niñas aún tienen una desproporcionada desventaja en su acceso a la educación, prerrequisito indispensable en el mundo moderno. Mientras que hoy más mujeres jóvenes reciben una educación básica (7 o más años (e escolaridad) que sus madres, las niñas en muchos países en desarrollo reciben menos escolaridad que los niños, y aquellas que residen en áreas rurales, reciben menos que las niñas en comunidades urbanas.

Actividad sexual y matrimonio: En gran parte del mundo la mayoría de las mujeres tienen su primera experiencia sexual en la adolescencia. La mujer que se casa en la adolescencia carece de educación, experiencia y de las habilidades que una mujer un poco mayor podría traer a su matrimonio y familia. Los niveles de unión y matrimonio tempranos han disminuido con respecto a la generación anterior, pero aún subsiste comúnmente en algunas regiones.

Uso de anticonceptivos: El uso de anticonceptivos en los adolescentes solteros y casados es más común que en el pasado, pero aún en gran parte del mundo su uso es bajo. La mayoría de las mujeres jóvenes ha oído hablar acerca de los modernos métodos anticonceptivos, pero posiblemente carece de acceso a ellos, no tiene información sobre su uso apropiado o no los usa porque se espera que tenga un hijo tan pronto se case.

Embarazo y nacimiento en la adolescencia: Los nacimientos en madres adolescentes están decreciendo en países donde era común a medida que el acceso a la educación aumenta y al amplio reconocimiento de las ventajas tanto en salud social como económicas- de retrasar los nacimientos.

Servicios de salud reproductiva para las mujeres jóvenes: Las adolescentes sexualmente activas se enfrentan a una variedad de riesgos que incluyen embarazos no deseados, abortos inseguros y enfermedades de transmisión sexual que atentan contra su salud sexual y reproductiva y que posiblemente afectan su fertilidad futura. Enfrentadas a un embarazo no deseado, algunas mujeres jóvenes buscan el aborto clandestino, el cual pone en riesgo su salud o su propia vida. Infecciones relacionadas con el aborto son especialmente comunes en los países donde el aborto está legalmente limitado y esto frecuentemente compromete una gran parte de los pacientes hospitalizados por complicaciones en estos procedimientos.

Las enfermedades de transmisión sexual incluido el VHI/SIDA son la mayor causa de infecciones del tracto reproductivo y atentan contra la fertilidad futura y la vida de las mujeres jóvenes y afecta la salud de los recién nacidos manifestándose en bajo peso al nacer, niños prematuros y aumento de la vulnerabilidad a infecciones y enfermedades.

Las relaciones sexuales producto de la fuerza, la coerción, el abuso y algunas prácticas culturales tales como la mutilación genital femenina, pone en peligro la salud reproductiva de la gente joven. Las mujeres jóvenes abusadas sexualmente están en riesgo de infección, de maternidad indeseada y pueden también sufrir otros traumas y aflicción psicológica.

"La sociedad debe preparar a sus jóvenes para enfrentar un mundo bastante diferente al de sus padres". 
Resumen de los retos revelados en este nuevo informe "Hacia un Nuevo Mundo".

"Ellos necesitan la guía y el apoyo de sus familias y comunidades, la atención de un gobierno comprometido con su desarrollo... la importancia de invertir en la vida de los jóvenes -el replanteamiento de políticas y prioridades comprometiendo recursos financieros e implementando programas- esto va más allá de los simples números. La adecuada manera como las comunidades y las naciones satisfagan estas necesidades de la gente joven, no sólo determinará la clase de vida de estos jóvenes, sino que también se extenderá a la vida de los niños que ellos traigan a este mundo y a las sociedades que ellos construirán y preservarán.

El Instituto Alan Guttmacher es una organización sin ánimo de lucro enfocada a la investigación de la salud reproductiva, al análisis de las políticas y de la educación pública con oficinas en Nueva York y Washington D.C.

Datos sociodemográficos de Colombia

Totales

- 7.551 .800 adolescentes (10-19 años) viven en Colombia y representan el $21 \%$ de la población del país.

- $\quad 1.832 .000$ de estos adolescentes son mujeres y jóvenes (15-19 años).

- 7 de cada 10 colombianos viven en áreas urbanas.

Educación y empleo

- Por cada 100 niños matriculados en la escuela secundaria, 116 mujeres están matriculadas.

- Sin embargo sólo el 63\% de mujeres de 15 a 19 años ha tenido 7 o más años de escolaridad.

- Hoy la mujer joven en Colombia está más dispuesta a alcanzar este nivel de educación que las generaciones anteriores.

- El 40\% de mujeres entre 18 y 19 años ha tenido más de 10 años de educación.

- El 28\% de mujeres entre 15 y 19 años está desempleada.

- Entre las mujeres jóvenes de 11 países estudiados de América Latina, las colombianas son las más dispuestas a tener un mejor nivel educativo (10 o más años de educación).

\section{Actividad sexual y matrimonio}

- El $62 \%$ de las mujeres entre 20 y 24 años inician su actividad sexual antes de los 20 -el $35 \%$ antes del matrimonio.

- El 14\% de las mujeres jóvenes están casadas o cohabitan, comparado con el 6\% de los hombres jóvenes entre 15 y 19 años que se han casado.

- El 26\% de las mujeres jóvenes inician su primer matrimonio o unión antes de los 18 años.
- Si una mujer joven tiene menos de 7 años de escolaridad, es casi tres veces más propensa a casarse o estar en unión a los 18: el 42\%, comparado con el $15 \%$ de mujeres jóvenes con mayor nivel educativo.

\section{Conocimiento y uso de anticonceptivos}

- Muchas mujeres jóvenes conocen acerca de la moderna anticoncepción. 97\% saben acerca de la píldora, inyectables o implantes. $96 \%$ conocen el condón.

- El 81\% saben dónde obtener un método moderno.

- El 30\% de las mujeres jóvenes casadas usan la píldora, inyectables, DIU o implante, un 4\% usa el condón y el 17\% usa otros métodos.

- El 16\% de las mujeres jóvenes solteras y sexualmente activas usan la píldora, inyectables, DIU o implante, 10\% usa el condón y 19\% otros métodos.

- Entre las mujeres jóvenes de los países de América Latina de los cuales se tienen datos, las colombianas están entre las que más seguramente están usando un método anticonceptivo moderno.

- Entre todas las mujeres jóvenes de 15 a 19 solamente el $8 \%$ usa un método moderno.

- Entre todas las mujeres de 15 a 19, el 8\% no usa anticonceptivos y el 3\% usa un método tradicional aunque están casadas o sexualmente activas y no desean tener un hijo pronto.

\section{Nacimientos}

- En 1997, 163.000 niños nacieron de mujeres entre los 15 y 19 años de edad. $9 \%$ de mujeres entre 15 a 19 años dan a luz cada año.

- El 36\% de mujeres de 20 a 24 dieron a luz a los 20 años de edad.

- Mujeres con 7 o más años de escolaridad son mucho menos propensas a tener un hijo antes de los 18 años: $8 \%$, comparado con el $33 \%$ de las mujeres jóvenes con menos de 7 años de escolaridad.

- El 44\% de todos los nacimientos en la adolescencia en Colombia no son planeados.

- Los nacimientos en la adolescencia apenas han cambiado en los últimos 20 años. Es tan probable que las mujeres entre los 20 y 24 años hayan tenido un hijo en la adolescencia como las mujeres de 40 o 44 años.

- Entre las mujeres jóvenes casadas, el 83\% no quiere tener hijos pronto.

- En América Latina, Colombia tiene una de las más altas tasas de nacimientos no planeados en la adolescencia $(31 \%)$.

- En promedio, las mujeres jóvenes en Colombia desean una familia de dos (2) hijos. 